\title{
Surgical site infections in a tertiary hospital over 10 years
}

\author{
The effect of hospital accreditation strategy implementation
}

Lateefah T. Alshammari, MBBS, Sarah A. Alkatheer, MBBS, Maymona B. AlShoaibi, MBBS, Aqdas A. Alomran, MBBS, Sharifah N. Almulhim, MBBS, Reem Y. Aljindan, MD, PhD, Yasser M. Aljehani, MD, Khaled R. Alkharsah, PhD.

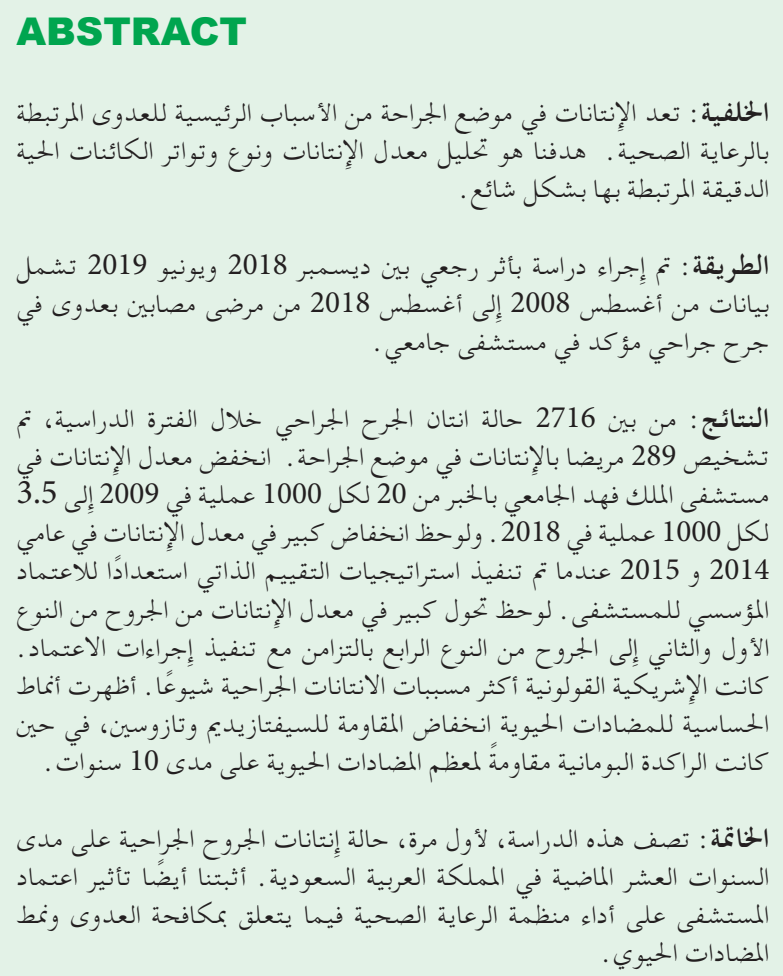

Objectives: To analyze the rate of surgical site infections (SSIs), the type, and the frequency of the commonlyassociated microorganisms.

Methods: A retrospective study was conducted in King Fahd Hospital of the University, Al Khobar, Saudi Arabia between December 2018 and June 2019 comprising data from August 2008 to August 2018 from patients with culture-confirmed surgical site infection at a tertiary hospital.

Results: Out of 2716 wound infection cases during the study period, a total of 289 patients were diagnosed with SSI. The rate of SSI in the tertiary hospital decreased from 20 per 1000 operations in 2009 to 3.5 per 1000 operations in 2018. A significant decrease in the rate of SSIs was observed in 2014 and 2015 when self-assessment strategies in preparation for the accreditation of the hospital were implemented. A significant shift in the SSI rate from type I and II wounds to type IV wounds was observed coinciding with implementation of accreditation procedures. Escherichia coli was the most common pathogen. Antibiotic susceptibility patterns showed reduced resistance to ceftazidime and tazocin, while Acinetobacter baumannii was resistant to most of the antibiotics over 10 years.

Conclusion: This study describes, for the first time, the status of SSI over the past 10 years in Saudi Arabia. The study also demonstrated the effect of hospital accreditation on healthcare organization performance regarding infection control and antibiogram pattern.

Keywords: infections; postoperative complications; surgical wound infection; wound infection; microbial sensitivity tests

Saudi Med J 2020; Vol. 41 (9): $971-976$ doi: 10.15537/smj.2020.9.25347

From the Department of Microbiology (Alshammari, Alkatheer, AlShoaibi, Alomran, Almulhim, Aljindan, Alkharsah) and from the Department of Surgery (Aljehani), College of Medicine, Imam Abdulrahman Bin Faisal University, Dammam, Kingdom of Saudi Arabia.

Received 7th June 2020. Accepted 19th August 2020.

Address correspondence and reprint request to: $D r$. Khaled R. Alkharsah, Department of Microbiology, College of Medicine, Imam Abdulrahman Bin Faisal University, Dammam, Kingdom of Saudi Arabia. E-mail:kalkharsah@iau.edu.sa

ORCID ID: https://orcid.org/0000-0002-4641-2604

Surgical site infections (SSIs) are defined as soft $\checkmark$ tissue, deep tissue, or organ infections that are observed within 30 days postoperatively or within one year in cases of foreign body implantation. They are considered as one of the most important causes of 
postoperative complications. The Centers for Disease Control and Prevention (CDC) classifies wound types into 3 groups each of which carries an expected risk of infection. ${ }^{1,2}$ Several previous studies found that the most common organisms causing surgical wound infections were Staphylococcus aureus (S. aureus) and the more antibiotic resistant strain methicillin-resistant $S$. aureus (MRSA)..$^{3-5}$ Additionally, the American College of Surgeons and Surgical Infection Society identified several factors that predispose for the development of SSI in their guidelines. ${ }^{6}$ These factors can be classified as either intrinsic (patient-related) or extrinsic (procedure-related). Risk factors such as age, diabetes, nutritional status, obesity, smoking, preoperative length of stay, coexistent infections, and colonization with microorganisms are considered patient-related. While factors related to procedures involve the duration of surgical scrubbing and duration of operation, skin antiseptic preparation, and shaving and prepping before operation. ${ }^{6,7}$ Furthermore, environmental factors such as fungal and bacterial colonization on surfaces and the quality of air in the operating rooms are associated with SSIs. ${ }^{8,9}$ The Physical Status Classification System of the American Society of Anesthesiologists links the higher rates of SSI with more unstable and critical patients. ${ }^{10}$ Other risk stratification strategies have been developed to predict the likelihood of occurrence of SSIs, such as the Surgical Site Infection Risk Score; ${ }^{11}$ however, a standardized strategy for all patients has not been determined. ${ }^{12,13}$

Hospital-acquired infections including SSIs can increase the length of stay (LOS) and cost of treatment resulting in poorer patient outcomes. Approximately $20 \%$ of hospital-acquired infections are caused by SSIs. ${ }^{14}$ Studies conducted in the United States and England have unanimously concluded that SSIs increase the LOS and overall hospital costs. ${ }^{15}$ The increase in LOS is dependent on the nature of the SSI, whether superficial or deep, and the type of procedure. ${ }^{15}$ On average, SSI resulted in an additional 4-13 days of hospital stay and a 3-fold increase in the likelihood of readmission. . $^{14,16}$

Timely administration of preoperative antibiotics is considered an important practice to prevent SSIs. ${ }^{17,18}$ Additionally, reducing the time of surgery and performing an open approach during surgery

Disclosure. Authors have no conflict of interests, and the work was not supported or funded by any drug institution. can reduce the risk of SSIs according to the recent guidelines advocate for the use of minimally invasive techniques. ${ }^{19,20}$ Other preventive measures include controlling patient's modifiable risk factors to enhance wound healing, preoperative preparation by smoking cessation, nutritional support, and controlling blood glucose. $^{20}$

We described in this report the frequency and rate of SSI at a tertiary hospital and identified the causative organisms associated with SSIs, along with their antimicrobial susceptibility pattern, over a period of 10 years. We also demonstrated the effect of implementing hospital accreditation strategies on the rate of SSIs.

Methods. This retrospective study was conducted between December 2018 and June 2019. The study included data from culture-confirmed surgical site infections in patients who underwent surgical intervention in King Fahd Hospital of the University (KFHU), Al Khobar, Saudi Arabia between August 2008 and August 2018. We employed the CDC definition and classification of surgical wound infections to define and classify our cases. ${ }^{2}$ The study was conducted in agreement with the principles of Helsinki Declaration. The ethical approval for the study was obtained from the Research Ethics Committee of Imam Abdulrahman Bin Faisal University (Number: IRB-UGS-2018-01-218).

Patients' demographic and clinical data were collected from the medical records.

Patients were selected if they had a record of a samples at the microbiology laboratory labeled with "wound swab," "abscess," "wound," "wound drain," "wound culture," "wound discharge," or any combination of these keywords from August 2008 to August 2018. Careful inspection of all selected patients' files was performed to exclude samples that do not fit the definition of SSI. Additionally, samples were excluded if the patients underwent surgical procedures in another hospital, if samples were inappropriately collected, duplication, or if the culture result suggested a normal skin flora.

Statistical analysis. The rate of SSI was calculated as the number of SSIs per 1000 operations per year. Because the data were not completely available for the years 2008 and 2018, the rates of SSI for these years were estimated based on the average total number of operations annually for the years 2009-2017. Descriptive analysis including frequencies and designing multivariate tables were performed using the software IBM SPSS Statistics for Windows, version 26 (IBM Corp., Armonk, N.Y., 
USA). Excel software from Microsoft office was used to design figures and graphs.

Results. A total of 2716 reads were obtained from the hospital records using the search words mentioned above. A total of 400 samples were excluded because they did not meet the CDC definition of SSI. Moreover, 2027 samples were excluded because of the following reasons: duplication (most common), surgery being performed in another hospital, mislabeling, missing data about infection, and contamination. A total of 289 samples of culture-confirmed SSIs were included in our study.

The rate of SSIs among females (55.6\%) was higher than that of males $(44.4 \%)$, but it was statistically insignificant $(p=0.06)$. There was a statistically significant difference in frequency of SSIs among males and females in different age groups (Chi-squared for linear trend [extended Hans-Mantel] $=4.6 ; p=0.03$ ) (Table 1).

The rate of SSIs decreased over the past 10 years from approximately 20 cases per 1000 operation in 2009 to 3.5 cases per 1000 operations in 2018 (Figure 1). The most significant decrease in the rate of SSIs was observed in 2014 and 2015 (Figure 1).

Almost one-fifth of the SSIs was observed in vascular surgeries $(20.85 / 1000)$, followed by general surgeries (12.18/1000), neurosurgeries (10.8/1000), orthopedic surgeries (7.48/1000), and obstetrics and gynecology surgeries $(5.35 / 1000)$.

Table 1 - Age groups and gender of patients diagnosed with surgical site infections in the study.*

\begin{tabular}{lccccc}
\hline Age & $\begin{array}{c}\text { Male } \\
\text { n }(\%)\end{array}$ & $\begin{array}{c}\text { Female } \\
\text { n }(\%)\end{array}$ & $P$-value $^{\dagger}$ & Total \\
\hline $0-10$ & $19(67.9)$ & $9(32.1)$ & 0.005 & $28(10.5)$ \\
$11-20$ & $12(57.1)$ & $9(42.9)$ & 0.11 & $21(7.9)$ \\
$21-30$ & $23(51.1)$ & $22(48.9)$ & 0.16 & $45(16.9)$ \\
$31-40$ & $12(26.7)$ & $33(73.3)$ & 0.004 & $45(16.9)$ \\
$41-50$ & $16(38.1)$ & $26(61.9)$ & 0.19 & $42(15.8)$ \\
$51-60$ & $16(37.2)$ & $27(62.8)$ & 0.15 & $43(16.2)$ \\
$>61$ & $20(47.6)$ & $22(52.4)$ & 0.32 & $42(15.8)$ \\
Total & $118(44.4)$ & $148(55.6)$ & & 266 \\
& & & & $(100 \%)^{\ddagger}$ \\
\hline
\end{tabular}

"Chi-squared for linear trend (extended Hans-Mantel) = 4.6, $p=0.03$; ${ }^{\dagger}$ Mid-P exact, ${ }^{\ddagger} 31$ samples were excluded in this analysis because they were duplicates from the same patients with different surgeries and SSIs over the 10-year study period
Additionally, our results showed that SSIs were observed more frequently in class I wounds representing $37 \%$ of cases, while class II $31 \%$, class IV $22 \%$, and class III wounds represented $12 \%$ of our sample. Escherichia coli were found to be more frequent in class IV and P. aeruginosa in class III wound types, whereas Enterococci and $A$. baumannii were the predominant organisms in class I wound category. From 2014 onward, SSIs rate decreased in class I and II wounds and became significantly lower than the rate in class IV wounds ( $p=0.025$ for class I and $p=0.034$ for class II wound) (Figure 2).

Escherichia coli was the most frequently isolated bacteria from SSIs over the study period (18.4\%). The second most commonly isolated bacteria were Pseudomonas aeruginosa (16.3\%), Klebsiella pneumoniae (9.6\%), and Acinetobacter baumannii (9.4\%).

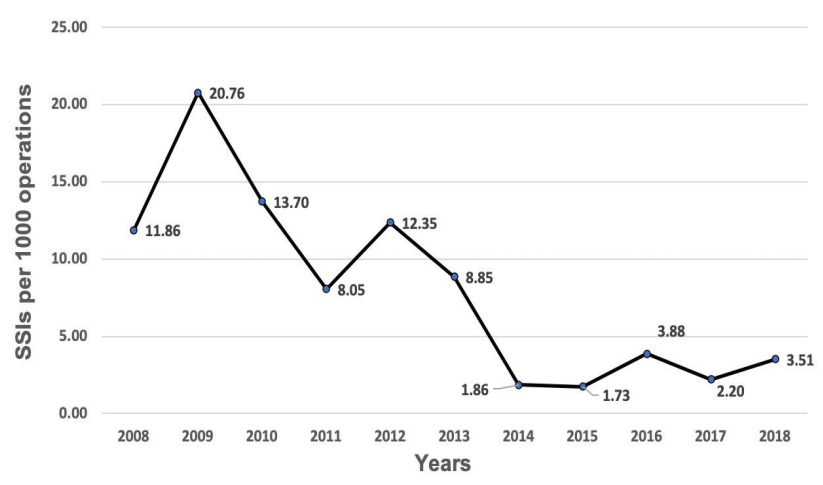

Figure 1 - The rate of surgical site infections (SSIs) over the past 10 years reported as the number of SSIs/1000 operations per year. The bars show the number of SSIs reported in each month. Because the data were not completely available for the years 2008 and 2018, the rates of SSI for these years were estimated based on the average total number of operations every year for the years 2009-2017.

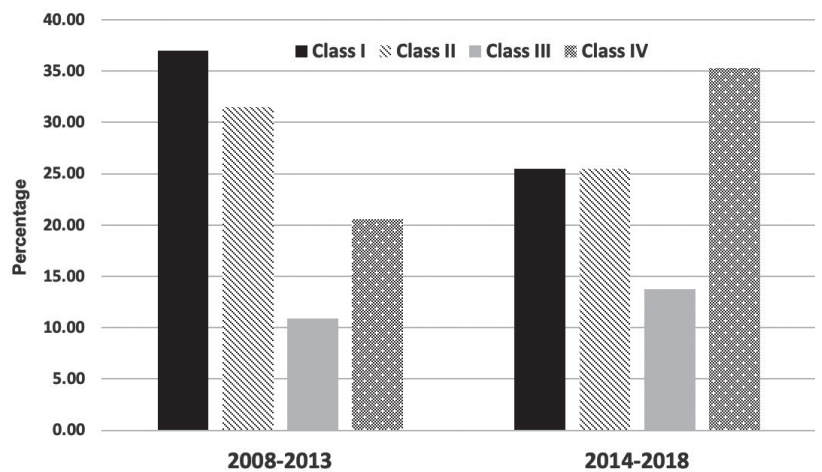

Figure 2 - Change in the surgical site infections rate based on the type of wound over the study period. 
Staphylococcus epidermidis and Enterobacter spp. had equal isolation frequency $(8.4 \%$ each), Enterococcus faecalis (7.3\%), and S. aureus (5.6\%) (Figure 3). Pseudomonas aeruginosa was the most common cause of SSIs in 2008 and 2009, and was less prevalent in the subsequent years (Figure 1). On the contrary, E. coli was less prevalent in the years 2008 and 2009 and became more frequently isolated from SSIs over the study period (Chi-squared for trend $p<0.0001$ ) (Figure 3). Other microorganisms such as Acinetobacter, S. aureus, Klebsiella spp., Enterococcus, and Enterobacter were consistently isolated from SSIs with variable frequencies (Figure 3).

Analysis of antibiograms of the most common organisms and antibiotics showed a change in the trend of antimicrobial resistance over the years (Figure 4). The resistance pattern of ceftazidime and tazocin decreased over time, while the resistance pattern of ciprofloxacin and Augmentin did not change over time (Figure 4).

Discussion. The rate of SSIs at our tertiary hospital decreased from approximately 20/1000 operations

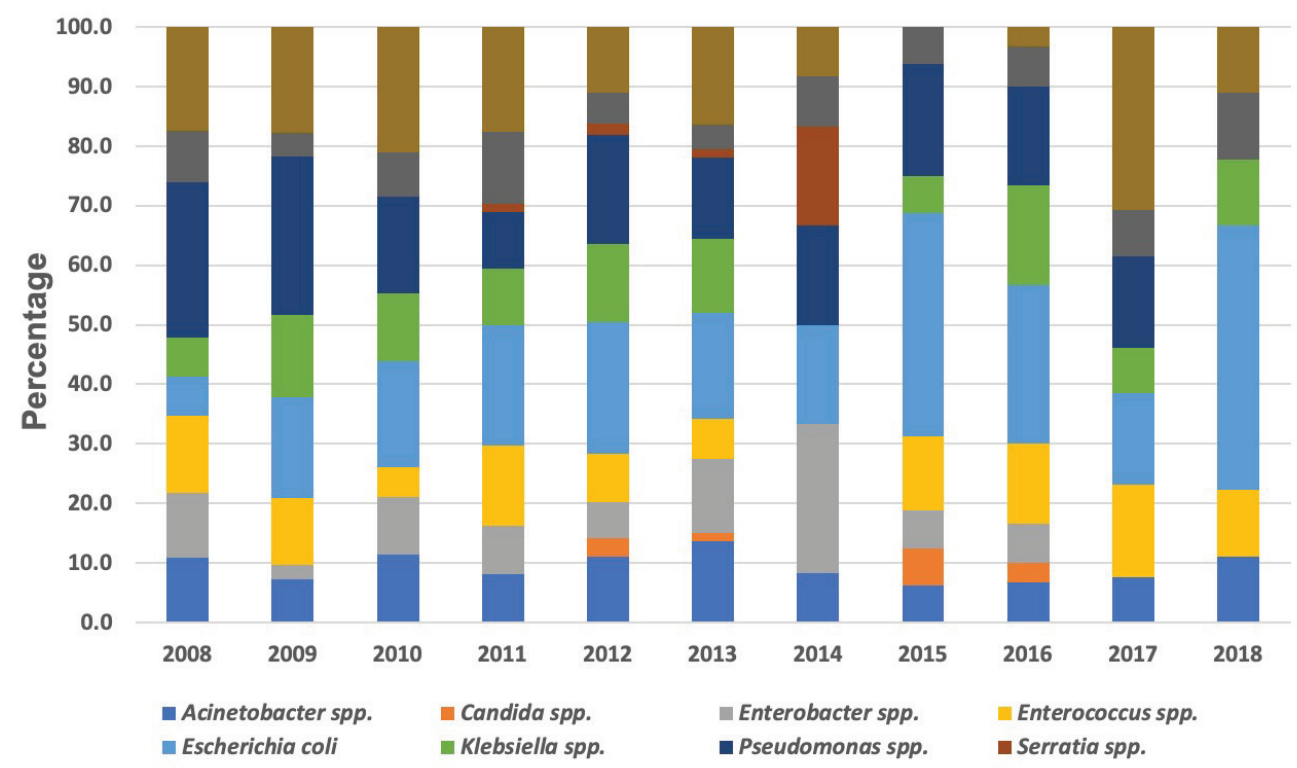

Figure 3 - Causative organisms isolated from surgical site infection cases over the study period.

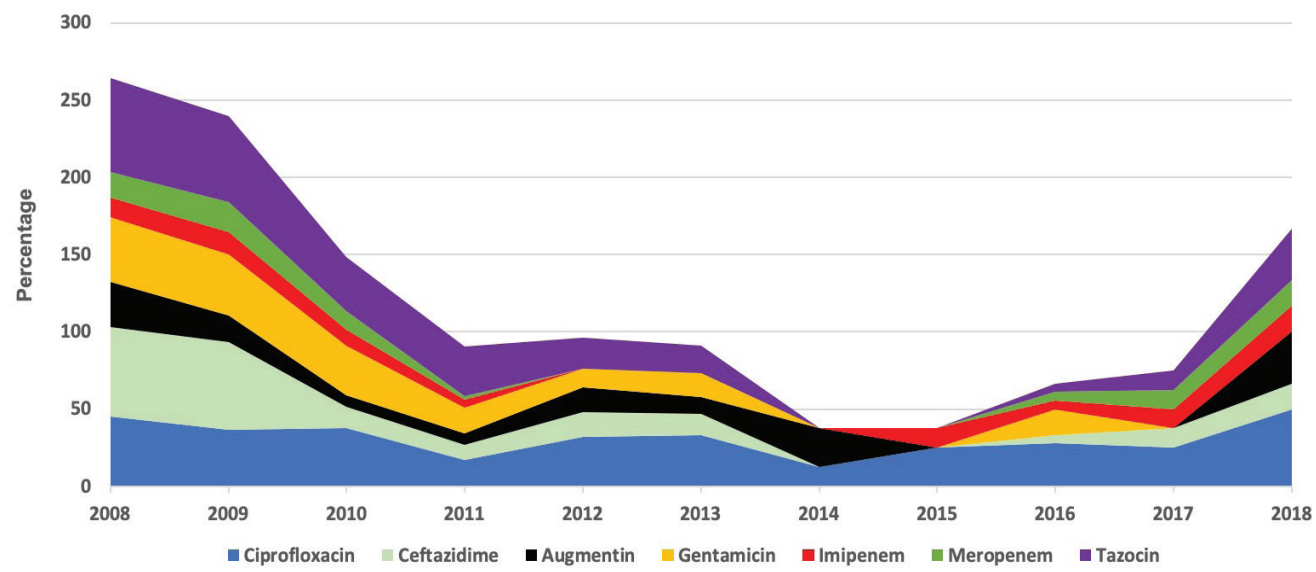

Figure 4 - General percentage of antimicrobial resistance patterns for the most commonly used antibiotics over the study period. 
in 2009 to $3.5 / 1000$ operations in 2018. The KFHU was granted Hospital Accreditation by the Joint Commission International (JCI) in September 2015. According to the JCI, the process of accreditations takes 18 to 24 months. $^{21,22}$ The process of hospital accreditation involves a self-assessment, revision, and analysis of standard procedures and their outcomes. This would explain the decrease in the rate of SSI in 2014 and 2015 and indicates the importance of such external peer assessment strategies on the quality of patients care.

In general, the female patients in this study had higher rate of SSIs than male patients aged between 30 and 40 years. This might be influenced by the fact that operations from the obstetrics and gynecology ward are included in the study, constituting 7\%-9\% of total operations annually. On the contrary, males aged less than 10 years have higher SSIs rate because this age group includes the over representation of males due to circumcision operations.

Several previous studies found that the most common organisms causing surgical wound infections were $S$. aureus and MRSA. ${ }^{4-6}$ However, according to our findings, Gram-negative bacteria were found to be the most common organisms including E. coli, P. aeruginosa, other members from the Enterobacteriaceae family.

Various factors play important roles in the risk of SSI occurrence, including lifestyle, comorbidities, medications, and length of hospital stay. ${ }^{13}$ Surgical wound classification has also been found to be a predictor of SSI risk. ${ }^{1,23}$ Previous studies assessing SSI found that SSIs are more commonly observed in class IV procedures. ${ }^{1}$ Furthermore and according to Mioton et al, ${ }^{1}$ the rate of SSI was similar for class I and II wounds at $0.64 \%$ and up to $2 \%$ for classes III and IV wounds. Conversely, the results of this study showed that SSI in KFHU was more common in classes I and II wounds than in class III and IV wounds. Nevertheless, in the years after 2014, a slight shift in the pattern of SSI was observed, where infections decreased in classes I and II to level lower than in class IV wounds. This also can be attributed to the strict application of scrubbing and disinfection through the implementation of accreditation policy and documentation procedures. The Annual European Epidemiological Report stated that the average percentage of SSI was $1.9 \%$, but varied depending on the procedure. ${ }^{24}$

Vascular surgery had the highest rate of SSI over the 10 years. It is consistent with the predictable rate of SSI according to the CDC considering that patients undergoing vascular surgery have more major risk factors for SSI than patients undergoing other types of surgeries. ${ }^{25}$ The second highest rate of SSI was observed in general surgery, with wound type II as the most common wound type. However, as of 2014, the rate of SSI after general surgeries started to decrease with wound type IV as the most common wound type, reemphasizing on the role of implementing accreditation strategies in the decreasing rate of SSI.

Antibiotic-resistance results in significant morbidity and mortality and is therefore a global concern. Unfortunately, the prevalence of these organisms is increasing among hospitalized patients, specifically in surgical and intensive care unit patients. ${ }^{26-28}$ Several factors can lead to the increase in antibiotic resistance, including antibiotic misuse and overuse. The reduction observed in microbial resistance to ceftazidime and tazocin in this study is interesting and is also consistent with the application of hospital accreditation policies.

Two of the most commonly encountered organisms in this study were $P$. aeruginosa and $A$. baumannii, both of which have shown significant resistance to some of the most potent antibiotics. According to the investigator's observation, patients frequently receive antibiotics for SSIs that have been proven to be resistant to that drug (data not shown). This issue is critical to patient care and may be attributed to the apparent lack of awareness among treating physicians. Mortality secondary to sepsis was identified in 7 patients in this study, 5 of which were due to carbapenem-resistant strains of $A$. baumannii. Al Jindan et $\mathrm{al}^{29}$ investigated this issue in KFHU and found that the organisms were most likely of the same nosocomial source.

We believe that our stringent criteria for filtering the samples and including only samples with most available data may have produced an underestimated rate of SSI at our hospital.

Study limitation. Most of the limitations in this study result from the lack of proper documentation of patient information and storage of results from diagnostic and treatment procedures at different sites, leading to discrepancies in the labeling of samples and swabs, missing data, and duplication of data.

In conclusion, this study describes, for the first time, the status of SSI over the past 10 years in Saudi Arabia. We also demonstrated the effect of hospital accreditation on healthcare organization performance regarding infection control and antibiogram pattern.

Acknowledgment. The authors are grateful to the IT department, the microbiology department, and the administration at King Fahd Hospital of the University, Al Khobar, Saudi Arabia for facilitating their work during data collection. The authors would like to thank the Editage (https://www.editage.com) for English language editing. 


\section{References}

1. Mioton LM, Jordan SW, Hanwright PJ, Bilimoria KY, Kim JY. The relationship between preoperative wound classification and postoperative infection: A multi-institutional analysis of 15,289 patients. Arch Plast Surg 2013; 40: 522-529.

2. Berrios-Torres SI, Umscheid CA, Bratzler DW, Leas B, Stone EC, Kelz RR, et al. Centers for Disease Control and Prevention Guideline for the prevention of surgical site infection, 2017. JAMA Surg 2017; 152: 784-791.

3. Weiss CA, 3rd, Statz CL, Dahms RA, Remucal MJ, Dunn DL, Beilman GJ. Six years of surgical wound infection surveillance at a tertiary care center: review of the microbiologic and epidemiological aspects of 20,007 wounds. Arch Surg 1999; 134: 1041-1048.

4. Ou L, Chen J, Hillman K, Flabouris A, Parr M, Assareh H, et al. The impact of post-operative sepsis on mortality after hospital discharge among elective surgical patients: a population-based cohort study. Crit Care 2017; 21: 34.

5. Eltahawy AT, Mokhtar AA, Khalaf RM, Bahnassy AA. Postoperative wound infection at a university hospital in Jeddah, Saudi Arabia. J Hosp Infect 1992; 21: 79-83.

6. Ban KA, Minei JP, Laronga C, Harbrecht BG, Jensen EH, Fry DE, et al. American College of Surgeons and Surgical Infection Society: Surgical Site Infection Guidelines, 2016 Update. J Am Coll Surg 2017; 224: 59-74.

7. Mangram AJ, Horan TC, Pearson ML, Silver LC, Jarvis WR. Guideline for Prevention of Surgical Site Infection, 1999. Centers for Disease Control and Prevention (CDC) Hospital Infection Control Practices Advisory Committee. Am J Infect Control 1999; 27: 97-132; quiz 3-4; discussion 96. Epub 1999/04/10.

8. Parvizi J, Barnes S, Shohat N, Edmiston CE, Jr. Environment of care: Is it time to reassess microbial contamination of the operating room air as a risk factor for surgical site infection in total joint arthroplasty? Am J Infect Control 2017; 45: $1267-1272$.

9. Alfonso-Sanchez JL, Martinez IM, Martin-Moreno JM, Gonzalez RS, Botia F. Analyzing the risk factors influencing surgical site infections: the site of environmental factors. Can J Surg 2017; 60: 155-161.

10. Isik O, Kaya E, Dundar HZ, Sarkut P. Surgical site infection: Re-assessment of the risk factors. Chirurgia (Bucur) 2015; 110 : 457-461.

11. van Walraven C, Musselman R. The surgical site infection Risk Score (SSIRS): A model to predict the risk of surgical site infections. PLoS One 2013; 8: e67167.

12. Anderson DJ, Podgorny K, Berrios-Torres SI, Bratzler DW, Dellinger EP, Greene L, et al. Strategies to prevent surgical site infections in acute care hospitals: 2014 update. Infect Control Hosp Epidemiol 2014; 35: 605-627.

13. Mueck KM, Kao LS. Patients at high-risk for surgical site infection. Surg Infect (Larchmt) 2017; 18: 440-446.

14. de Lissovoy G, Fraeman K, Hutchins V, Murphy D, Song D, Vaughn BB. Surgical site infection: incidence and impact on hospital utilization and treatment costs. Am J Infect Control 2009; 37: 387-397.
15. Schweizer ML, Cullen JJ, Perencevich EN, Vaughan Sarrazin MS. Costs associated with surgical site infections in Veterans Affairs Hospitals. JAMA Surg 2014; 149: 575-581.

16. Jenks PJ, Laurent M, McQuarry S, Watkins R. Clinical and economic burden of surgical site infection (SSI) and predicted financial consequences of elimination of SSI from an English hospital. J Hosp Infect 2014; 86: 24-33.

17. Classen DC, Evans RS, Pestotnik SL, Horn SD, Menlove RL, Burke JP. The timing of prophylactic administration of antibiotics and the risk of surgical-wound infection. $N$ Engl J Med 1992; 326: 281-286.

18. Bratzler DW, Hunt DR. The surgical infection prevention and surgical care improvement projects: national initiatives to improve outcomes for patients having surgery. Clin Infect Dis 2006; 43: 322-330.

19. Gandaglia G, Ghani KR, Sood A, Meyers JR, Sammon JD, Schmid M, et al. Effect of minimally invasive surgery on the risk for surgical site infections: results from the National Surgical Quality Improvement Program (NSQIP) Database. JAMA Surg 2014; 149: 1039-1044.

20. Leaper DJ, Edmiston CE. World Health Organization: global guidelines for the prevention of surgical site infection. $J$ Hosp Infect 2017; 95: 135-136.

21. JCI. Joint Commission International Accreditation Standards for Hospitals. 5th ed. Joint Commission International; 2013. $72 \mathrm{p}$.

22. JCI. Pathway to JCI Accreditation. [cited 2019 November 20th, 2019]; Available from: https://www. jointcommissioninternational.org/accreditation/pathway-toaccreditation/.

23. Onyekwelu I, Yakkanti R, Protzer L, Pinkston CM, Tucker C, Seligson D. Surgical wound classification and surgical site infections in the orthopaedic patient. J Am Acad Orthop Surg Glob Res Rev 2017; 1: e022.

24. ECDC. European Centre for Disease Prevention and Control. Annual Epidemiological Report 2016 - Surgical site infections. 2016.

25. Bandyk DF. Vascular surgical site infection: risk factors and preventive measures. Semin Vasc Surg 2008; 21: 119-123.

26. Aljindan R, Bukharie H, Alomar A, Abdalhamid B. Prevalence of digestive tract colonization of Carbapenem-resistant Acinetobacter baumannii in hospitals in Saudi Arabia. J Med Microbiol 2015; 64 (Pt 4): 400-406.

27. Pellegrini CA. Antibiotic resistance causing issues for surgical patients. Bull Am Coll Surg 2016; 101: 72-73.

28. Alam MZ, Alam Q, Jiman-Fatani AA, shukri HA, Haque A. A surveillance study on the prevalence and antimicrobial resistance pattern among different groups of bacteria isolated from Western province of Saudi Arabia. Biomedical Research 2017; 28: 898-906.

29. Aljindan R, Alsamman K, Elhadi N. ERIC-PCR Genotyping of Acinetobacter baumannii isolated from different clinical specimens. Saudi J Med Med Sci 2018; 6: 13-17. 